\title{
Optimisation techniques for planning automatic under frequency load shedding in New Zealand's power system
}

\author{
Robert Burdett $^{1} \quad$ Conrad Edwards $^{2} \quad$ John Boland $^{3}$
}

(Received 10 September 2015; revised 11 May 2016)

\begin{abstract}
Electricity generation is vital in developed countries to power the many mechanical and electrical devices that people require. Unfortunately electricity generation is costly. Although electricity can be generated easily but not normally stored. Electricity generation is also difficult to manage because exact demand is unknown from one instant to the next. A number of services are required to manage fluctuations in electricity demand, and to protect the system when frequency falls below acceptable levels. A current approach is called automatic under frequency load shedding. This article proposes new methods for optimising this approach in New Zealand's power system. The core ideas were developed during the 2015 Maths and Industry
\end{abstract}

http://journal.austms.org.au/ojs/index.php/ANZIAMJ/article/view/10015 gives this article, (c) Austral. Mathematical Soc. 2016. Published May 27, 2016, as part of the Proceedings of the 2015 Mathematics and Statistics in Industry Study Group. ISSN 1445-8810. (Print two pages per sheet of paper.) Copies of this article must not be made otherwise available on the internet; instead link directly to this URL for this article. 
Study Group in Brisbane, Australia. The problem was motivated by Transpower Limited, a company that manages New Zealand's power system and transports bulk electricity from where it is generated to where it is needed. The approaches developed in this article can be used in electrical power systems anywhere in the world.

\section{Contents}

1 Introduction

1.1 Research Approach . . . . . . . . . . . . . . . .

2 Research Trends

3 Original Formulation

M10

4 Reductions

M12

4.1 First Model Reduction . . . . . . . . . . . . . . . . . . M12

4.2 Second Model Reduction . . . . . . . . . . . . . . . . M16

4.3 Data Reduction . . . . . . . . . . . . . . . . . . M19

4.3.1 Stratified Sampling . . . . . . . . . . . . M19

4.3 .2 Ranking Feeders . . . . . . . . . . . . . M22

5 Bi Objective Model

M24

5.1 Model and Methodology . . . . . . . . . . . . . M24

5.2 Preliminary Results . . . . . . . . . . . . . . . . . . M28

5.3 Pareto Analysis . . . . . . . . . . . . . . . . . . . M28

6 Solution Techniques

M30

6.1 Optimisation Simulation . . . . . . . . . . M30

6.2 Meta Heuristic Approach . . . . . . . . . . . . . . . M31

6.3 Heuristic Approach . . . . . . . . . . . . . . . . M33

6.4 Solution Similarity and Repeatability .......... . M34 


\section{Introduction}

Electricity generation is vital in developed countries to power the many mechanical and electrical devices needed in homes, businesses and in heavy industry. This article considers issues encountered in New Zealand's power system. In that country about $75 \%$ of the electricity is created from renewable energy, like hydro (60\%), geothermal (10\%) and wind (5\%). Around $90 \%$ of the total electricity generated passes through the high voltage transmission system that spans the country; it is known as the national grid. Five generation companies, one transmission company and 33 distribution companies currently exist. The company that manages New Zealand's power system and transports bulk electricity from where it is generated, to where it is needed, is called Transpower Limited.

In New Zealand the electricity network is a radial (tree) system. Figure 1 summarises this system. This network delivers electricity at high voltage to substations (i.e., exit grid point) in each part of the country. Large cities may have several substations serving them, and in a few cases, electricity is directly supplied to large industrial consumers. Local distribution systems take the power delivered to each substation and divert it at lower voltages to homes and businesses. The locations where electricity passes from the national grid to local networks are called grid exit points (GXP). They signify the demarcation between generation and transmission with distribution and consumption. In New Zealand there are different electricity demands in different regions. These demands fluctuate during the day, over the course of a year and in different seasons. Each region contains one or more GXP.

At present electrical power generation is costly and difficult to manage because 
Figure 1: Electricity generation in New Zealand.

\section{The electricity supply chain in New Zealand}

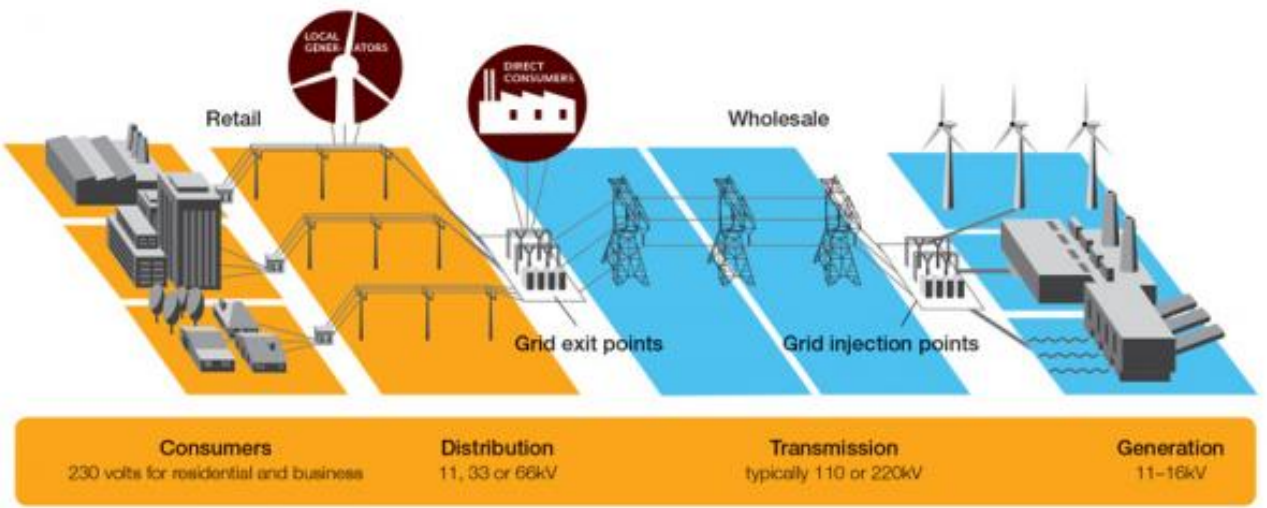

demand is unknown from day to day, and from one instant to the next, and electricity can be generated easily but not normally stored [4]. In New Zealand's power system the frequency is $50 \mathrm{~Hz}$. The acceptable range for this frequency is $47-52 \mathrm{~Hz}$. If the frequency falls below acceptable levels, then generators are programmed to automatically disconnect to protect equipment. The frequency can drop to $47 \mathrm{~Hz}$ within two-three seconds. Twenty four per cent of generators will trip (automatically disconnect) at $47 \mathrm{~Hz}$. In the worst case, a national blackout may occur when electricity supply is permanently disrupted. In a normal working day, generation output is readjusted every five minutes to meet customer needs.

The importance of reserves is specified by Lopes et al. [7] and Wong \& Fuller [9]. For example, Wong \& Fuller [9] stated that reserves must be present in the bulk electric power system so that the operator can replace or redistribute generation, on short notice, in the event of the failure of equipment. Lopes et al. [7] stated that reserve energy is required to provide rapid access to generation, to accommodate errors in demand forecasting, to provide contingency arrangements for generation failures and to restore 
frequency response capabilities.

In New Zealand a number of services are incorporated to manage fluctuations in electricity demand.

- Frequency keeping: This is done by generators. They can quickly change output to match load fluctuations.

- Instantaneous Reserve: Spare generating capacity is used to cover potential events.

- Over Frequency Reserve: Generation is reduced to prevent an unplanned rise in frequency

- Voltage Support: Voltage is increased or decreased when and where needed

Automatic under frequency load shedding (AUFLS) is a strategy for handling uncertain events that threaten security of the system. AUFLS is a last resort and has been created in order to prevent a system collapse when the frequency rises or falls beyond specified tolerances. In essence, AUFLS is a mechanism by which large blocks of load are shed to prevent a system collapse. This is done by placing voltage-sensitive relays on load feeders around the system. AUFLS is also commonly referred to as extended reserves. Overseas systems have more AUFLS blocks that are smaller in size [10]. This allows more control to manage the frequency after an event. New Zealand's AUFLS scheme is made up of two $16 \%$ blocks. The extended reserves technical working group (ERTWG) was established to investigate and trial proposed changes to the AUFLS system.

\subsection{Research Approach}

This article considers the optimisation of AUFLS and in particular the placement and arming of relays The two decision problems considered at the 
Table 1: key terms used in this article.

\begin{tabular}{|c|c|}
\hline Term & Description \\
\hline Block & A measure of demand (i.e., load). \\
\hline Frequency & Rate of cyclic change in value of current and voltage. \\
\hline (power) & Measure of frequency is Hertz, i.e., one cycle per second. \\
\hline $\begin{array}{l}\text { Frequency } \\
\text { control }\end{array}$ & $\begin{array}{l}\text { Adjustment of power generation to maintain system fre- } \\
\text { quency close to the target frequency. }\end{array}$ \\
\hline Feeder & A circuit which provides a direct connection to a customer. \\
\hline VoLL & Value of lost load. \\
\hline Relay & Device to regulate frequency. \\
\hline $\begin{array}{lr}\text { Load } & \text { shed- } \\
\text { ding } & \text { and } \\
\text { control } & \end{array}$ & $\begin{array}{l}\text { The forced disconnection of load in stages (manual or } \\
\text { automatic). Forms of control include automatic under } \\
\text { frequency load shedding, interruptible load, manual load } \\
\text { shedding. }\end{array}$ \\
\hline $\begin{array}{l}\text { Interruptible } \\
\text { load }\end{array}$ & $\begin{array}{l}\text { Demand which, by commercial arrangement between the } \\
\text { grid operator and a provider, may be disconnected without } \\
\text { prior warning for the purposes of grid security. It triggers } \\
\text { at } 49.2 \mathrm{~Hz} \text { and takes up to one second to respond. }\end{array}$ \\
\hline AUFLS & $\begin{array}{l}\text { Automatic frequency load shedding. Block } 1 \text { triggers at } \\
47.8 \mathrm{~Hz} \text { and takes } 0.4 \text { seconds to respond. Power supply is } \\
\text { disconnected to } 16 \% \text { of customers across the island. AUFLS } \\
\text { Block } 2 \text { triggers at } 47.5 \mathrm{~Hz} \text { and a further } 16 \% \text { of customers } \\
\text { are disconnected. }\end{array}$ \\
\hline
\end{tabular}


Mathematics in Industry Study Group (MISG) are named "Relay Placement" and "Relay Arming". These are explained briefly below:

- Relay Placement: In which feeders should relays be placed in order to minimise a cost objective.

- Relay Arming: Which relays should be armed to minimise a cost objective.

In these problems the cost of non-supply is very important. It is commonly referred to as the value of lost load. The cost of placing and arming relays is a dominant concern. A number of physical requirements also must be met. To our knowledge the decision problems are not non-deterministic polynomial time hard (NP-hard), but they are large. Transpower indicated that the second problem is comparatively simpler to solve. In the past Transpower solved these problems using integer programming formulations. However, it has been reported that standard solvers such as CPLEX cannot find global optimal solutions quickly, if at all. Obtained solutions are not always the same, although their cost differences may be minimal. There is no constraint on solution time; however, improvements on current computing requirements are welcome and desirable. In response the group at MISG took a four stage approach consisting of the following tasks

1. model analysis,

2. analysis of decision making criteria,

3. analysis of solution techniques, and

4. data analysis and data reduction.

Model reduction and reformulation was first considered as that is the primary source of the difficulties encountered in practice (Sections 3-4). Solution robustness and repeatability, multiple objectives and simulation were then considered (Section 5). As a means of solving the decision problems, heuristics, meta-heuristics, column generation, constraint programming and decomposition techniques were investigated (Section 6). The data provided by 
Transpower was analysed and data reduction strategies were investigated last.

\section{Research Trends}

Power systems have been a topic of research for many years. This section discusses recent trends but is not a comprehensive list of all work that has been done. Thompson [8] reviewed the usage of voltage control relays when there is embedded generation. Thompson reported that as the loads connected to a distribution network vary throughout the day, so do the voltage drops. Embedded generation causes the reduction, or possibly reversal, of real power flow through upstream transformers. With increasing levels of distributed generation, the control of voltage levels across distribution networks requires a system that functions under dynamic operating conditions. Hiscock et al. [3] described a voltage control methodology that they developed. Load Drop Compensation (LDC) is a technique used to offset these voltage drops across a network caused by load current, where the target voltage of the regulator is adjusted such that the load is delivered at the optimum voltage level. Kusano [6] discussed protection relay and substation automation system. It describes the status of ongoing technical activities, as well as background circumstances, in protection and control systems in Japan, to ensure stable power supply, improved efficiency, and reduced cost. The protection of the natural environment at the same time is also required. Approaches to improve the reliability in the distribution substation are presented, followed by the anticipated effects of introducing distributed generation systems into the distribution networks. Just \& Weber [4] considered the pricing of reserves. They report that efficient dispatching and scheduling decisions cannot be made in real-time and that real-time generation, dispatch and demand adjustments are required because electricity cannot be stored. Spot markets, with the main function of efficiently dispatching the system, are held some time ahead of physical energy delivery and dispatch, typically days or hours ahead in most liberalized markets. Dispatching decisions also have to be made some 
time before actual delivery. In practice power plant as well as transmission line failures might occur and forecasts of load and intermittent supply are inevitably inaccurate. Hence, the necessary real-time adjustment can only be achieved by the provision and use of flexible system reserve capacity.

Wong [9] presented a stochastic linear programming model that can be used for pricing in electrical energy and reserve markets. It addresses capacity, energy, and reserve dispatch problems. Several possible ways to price energy and reserves were examined. Their approach is based on a new method for the joint dispatch of energy and reserves. An important feature is the ability to take into consideration single contingencies when determining optimal dispatch. El-Khattam et al. [5] addressed optimal investment planning for distributed generation within a competitive electricity market. They proposed a new optimization model and heuristic approach for distributed generation (DG) capacity investment planning. Optimal sizing and siting decisions for DG capacity is obtained through a cost-benefit analysis. The proposed heuristic method helps alleviate the use of binary variables in the optimization model thus easing the computational burden substantially. The developed framework calculation is based on benefit cost ratio (BCR) criteria. The analysis considers two main policy scenarios:

1. the disco is contracted to buy a fixed amount of power from a generating company through a bilateral contract with fixed price and has therefore no direct link with market price fluctuations; and

2. the disco operates as a competitive market participant and its capacity investment decisions are affected by market price fluctuations and investment budget constraints.

Arroyo \& Galiana [1] analysed pricing issues in security-constrained electricity markets that are subject to transmission flow limits. They formulated a general market-clearing process as an optimization problem. It accounts for transmission flow limits, preventive and corrective security, unit commitment, and two types of reserves offered by both generators and loads. They conclude that only products whose marginal costs can be separately and uniquely 
defined and calculated are those of energy and security at each node. Thus, under marginal pricing, all scheduled reserve types should be priced not at separate rates but at a common rate equal to the marginal cost of security. Arroyo \& Galiana [1] reported that when transmission flow limits are active, the scheduling of nodal reserves to provide sufficient flexibility to survive a set of credible contingencies is a difficult problem.

\section{Original Formulation}

The original formulation for the selection decision problem is first discussed. The main idea of this model is how to position AUFLS demand units over time within AUFLS blocks. An AUFLS demand unit means a block of demand (including a feeder or group of feeders) which can be automatically disconnected using an AUFLS system. Index $j$ is used to describe particular demand units (i.e., feeders). The index for blocks is b and the set of blocks is B. Time periods are denoted by index $t$. In addition an AUFLS region is defined. This is a group of connection locations. Each AUFLS region has an AUFLS requirement. This is the total amount of demand that must be able to be disconnected by the operation of all automatic under frequency load shedding systems in the AUFLS region. Regions are described by index $z$.

Assumption: When a demand unit is assigned to a block then it means that the demand unit has a relay placed on it.

According to Transpower [11] the flexible categorisation means that the demand unit can be armed/disarmed within seven days of receiving an instruction. The inflexible category means that the unit is permanently assigned to block $b$. 
The model is to minimise

$$
\begin{aligned}
& \text { totalcost }=\sum_{j} \sum_{b}\left[\left(\operatorname{rop}_{j}+\operatorname{rcp}_{j, b}+\operatorname{rfrp}_{j}+\text { duip }_{j, b}-\operatorname{frc}_{j}\right) \times \operatorname{inflex}_{j, b}\right] \\
& +\sum_{j} \sum_{b}\left[\left(\operatorname{rop}_{j}+\operatorname{rcp}_{j, b}+\operatorname{rfr}_{j}+\operatorname{rfp}_{j}\right) \times \operatorname{flex}_{j, b}\right] \\
& +\sum_{j} \sum_{b}\left[\left(\text { duip }_{j, b}-\text { frc }_{j}\right) \times \text { flex }_{j, b} \times\left(\frac{\text { flexProp }}{\text { flexProp }+ \text { addFlex }}\right)\right] \\
& +\sum_{j} \sum_{\mathrm{b}}\left[\frac{100 \times \text { regionPen }_{z, \mathrm{p}} \times \operatorname{regdev}_{\mathrm{t}, z, \mathrm{p}} \times \text { regionProp }_{z}}{\text { samplerate }}\right] \\
& +\sum_{j} \sum_{b}\left[\frac{100 \times \text { blockPen }_{z, b, p} \times \text { blockdev }_{t, z, b, p} \times \text { regionProp }_{z}}{\text { samplerate }}\right] \\
& +\sum_{j} \sum_{\mathrm{b}}\left[\frac{100 \times \text { flexPen }_{z, \mathrm{~b}, \mathrm{p}} \times \text { flexdev }_{\mathrm{t}, z, \mathrm{~b}, \mathrm{p}} \times \text { regionProp }_{z}}{\text { samplerate }}\right] .
\end{aligned}
$$

subject to, for all $t, z, b, p$ as appropriate,

$\sum_{b}\left(\right.$ flex $\left._{\mathbf{j}, \mathrm{b}}+\operatorname{inflex}_{\mathbf{j}, \mathbf{b}}\right) \leqslant 1, \quad$ for all $\mathfrak{j}$,

$\operatorname{regdev}_{\mathrm{t}, z, \mathfrak{p}} \geqslant \operatorname{sign}_{\mathrm{p}} \times\left(\operatorname{regionTar}_{z, \mathrm{p}}-\frac{\sum_{\mathrm{b}} \sum_{\mathrm{j} \in \operatorname{Regz}_{\mathrm{z}}} \text { unitMW }_{\mathrm{t}, \mathrm{j}} \times \operatorname{tot}_{\mathrm{j}, \mathrm{b}}}{\operatorname{regionDem}_{\mathrm{t}, \mathrm{z}}}\right)$,

$\operatorname{blockdev}_{t, z, b, p} \geqslant \operatorname{sign}_{\mathfrak{p}} \times\left(\operatorname{blockTar}_{z, b, p}-\frac{\sum_{j \in \operatorname{Regz}_{2}} \text { unitMW }_{t, j} \times \operatorname{tot}_{j, b}}{\operatorname{regionDem}_{t, z}}\right)$,

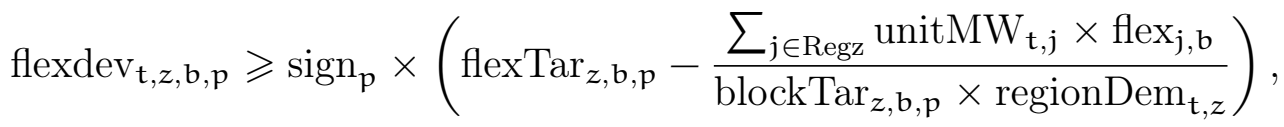

where tot ${ }_{j, b}=$ flex $_{j, b}+$ inflex $_{j, b}$. The objective is to minimize the total cost. The objective function includes penalty costs for deviating from target values. The parameters in the objective function are as follows: relay operating payment (rop), relay capital payment (rcp), relay flexibility payment (rfp), 
relay fast response payment ( $(\mathrm{frp})$, fast relay cost (frc), and demand unit interruption payment (duip). Constraint (2) ensures that only a single block is assigned for demand unit $\mathbf{j}$. Constraints (3), (4) and (5) are imposed in order to indirectly calculate deviations to the specified target levels. The index $p$ refers to situations where values are below the target lower bound, below the target, above the target, or above the target upper bound. The $\operatorname{sign}_{\mathrm{p}}$ parameter takes the following values $(1,1,-1,-1)$ respectively.

Suggestion There are minimum and maximum values for each specified target level. These should be added as constraints. Once added the number of solutions in the decision space should be greatly reduced and should facilitate a faster solve.

\section{Reductions}

\subsection{First Model Reduction}

In theory the original model could be simplified by replacing the existing binary variables (i.e., $f \operatorname{ex}_{\mathfrak{j}, \boldsymbol{b}}$ and $\operatorname{inflex}_{\mathfrak{j}, \boldsymbol{b}}$ ) with a reduced number of integer decision variables.

Proposed Transformation Let flex $\boldsymbol{j}_{j}$ and inflex $_{j}$ be the block assigned to demand unit $\boldsymbol{j}$. In other words, flex , inflex $_{\mathbf{j}} \in \mathrm{B}=\{0,1,2,3,4\}$ and $0 \leqslant$ flex $_{j} \leqslant|B|, 0 \leqslant$ inflex $_{j} \leqslant|B|$, where block 0 is a dummy block that refers to a non-assignment to any block.

The relationship between the old variables and the new ones is shown in the following property. 
Property 1 flex $_{j}=\sum_{b \in B}\left(b \times f l e x_{j, b}\right)$, and inflex $j_{j}=\sum_{b \in B}\left(b \times \operatorname{inflex}_{j, b}\right)$. In total the number of binary variables is $2|J||B|$, but the number of integer variables is much reduced, only $2|J|$. In total the search space for the binary variables $2^{2|J||\mathrm{B}|}=4^{|\mathrm{J}||\mathrm{B}|}$ but the search space for the integer variables is $|\mathrm{B}|^{|\mathrm{J}|}|\mathrm{B}|^{|J|}=|\mathrm{B}|^{2}|\mathrm{~J}|$. Define the ratio of the two as ratio $=4^{|\mathrm{J}||\mathrm{B}|} /|\mathrm{B}|^{2|\mathrm{~J}|}=$ $\left(4^{|\mathrm{B}|} /|\mathrm{B}|^{2}\right)^{|\mathrm{J}|}$. When $|\mathrm{B}|=5$ then ratio $=40.96^{\mid \mathrm{JJ}}$. This ratio increases greatly as the number of feeders increases and shows that the decision space is much smaller when integer variables are used. However, if this approach is to be successful, then the model constraints must be translated correctly. To date, two constraints have been successfully converted, but two have not. Exact details will be presented in due course. First, several important linearisations are developed.

Condition 1 If $x>0$ then $y=1$. If $x=0$ then $y=0$. Equivalent Constraints: $x \leqslant M y$ and $x \geqslant 1+M(y-1)$.

Proof: Let $y=0$. Therefore, $-M \leqslant x \leqslant 0 \Rightarrow x=0$ as $x \geqslant 0$ is already imposed. Let $y=1$. Therefore, $1 \leqslant x \leqslant M \Rightarrow x>0$.

Condition 2 If $x>0$, then $y=0$. If $y>0$, then $x=0$.

- Equivalent Condition $\neg(x>0 \wedge y>0)$.

- Equivalent Non Linear Constraint: $x y=0$.

- Equivalent Linear Constraints: $x>\gamma, x \leqslant M \gamma, \gamma+\frac{y}{M} \leqslant 1$.

Proof: Let $\gamma=1$ if $x>0$. Therefore $\gamma=0$ if $x=0$. If $\gamma=1$ then the first two constraints ensure that $1 \leqslant x \leqslant M$. If $\gamma=0$ then the first two constraints ensure that $x=0$. In the third constraint, if $\gamma=1$ then 
$y \leqslant 0 \Rightarrow y=0$ as $y \geqslant 0$ is already imposed. If $\gamma=0$ then $x=0$ and $y \leqslant M$ as required.

Condition 3 If $x=b$, then $y=1$. If $x \neq b$, then $y=0$. Equivalent Constraints: Not possible. A binary parameter is absolutely necessary. For example, if $x=b$, then $y_{b}=1$, else $y_{b}=0$.

The reformulation of the constraints is now discussed.

Constraint $1 \quad \sum_{b}\left(f l e x_{j, b}+\operatorname{inflex}_{j, b}\right) \leqslant 1$ for all $j$. Constraint Reformulation: this constraint is equivalent to $\mathrm{flex}_{j} \cdot \operatorname{inflex}_{j}=0$ as this equation allows both variables to be assigned no block, and stops both being assigned blocks simultaneously. This is equivalent to Condition 2. The following constraints are hence required and involve the introduction of binary variable $\gamma_{j}$ :

$$
\begin{aligned}
& \text { flex }_{j} \geqslant \gamma_{j} \text { for all } j ; \\
& \text { flex }_{j} \leqslant M \gamma_{j} \text { for all } j ; \\
& \gamma_{j}+\frac{\text { inflex }_{j}}{M} \leqslant 1 \quad \text { for all } j .
\end{aligned}
$$

\section{Constraint 2}

$$
\begin{aligned}
\operatorname{regdev}_{\mathbf{t}, z, p} & \geqslant \operatorname{sign}_{\mathfrak{p}} \\
& \times\left[\operatorname{regionTar}_{z, p}-\frac{\sum_{\mathbf{j} \in \operatorname{Reg} z} \sum_{\mathrm{b}} \text { unitMW }_{\mathrm{t}, \mathfrak{j}}\left(\text { flex }_{\mathbf{j}, \mathrm{b}}+\operatorname{inflex}_{\mathbf{j}, \mathrm{b}}\right)}{\operatorname{regionDem}_{\mathrm{t}, \boldsymbol{z}}}\right] .
\end{aligned}
$$

Constraint Reformulation: an equivalent condition in the new model is unitMW $_{t, j} \times \sum_{b}$ if $\left(\right.$ tot $\left._{j}=b, 1,0\right)$ where tot ${ }_{j}=$ flex $_{j}+$ inflex $_{j}$ which is now the index of a block. When summed over all blocks $b \mid b>0$, this condition may be written as $\sum_{j \in \text { Regz }}$ unitMW $_{t, j} \times \mathbf{i f}\left(\right.$ tot $\left._{j}>0,1,0\right)$. This "if" function 
is equivalent to Condition 1 as previously described. A binary variable $\alpha_{j}$ is introduced and the following constraints are added:

$$
\begin{aligned}
\operatorname{regdev}_{h, z, p} & \geqslant \operatorname{sign}_{\mathfrak{p}} \times\left(\operatorname{region}_{\operatorname{Tar}_{z, p}}-\frac{\sum_{j \in \operatorname{Reg} z} \text { unitMW }_{h, j} \alpha_{j}}{\operatorname{regionDem}}\right) ; \\
\operatorname{tot}_{j} & \leqslant M \alpha_{j} \quad \text { for all } j ; \\
\operatorname{tot}_{j} & \geqslant 1+M\left(\alpha_{j}-1\right) \text { for all } j .
\end{aligned}
$$

\section{Constraint 3}

blockdev $_{\mathrm{t}, z, \mathrm{~b}, \mathrm{p}} \geqslant \operatorname{sign}_{\mathrm{p}}$

$$
\times\left[\text { blockTar }_{z, b, p}-\frac{\sum_{\mathbf{j} \in \operatorname{Reg} z} \text { unitMW }_{\mathrm{t}, \mathrm{j}}\left(\text { flex }_{\mathbf{j}, \mathrm{b}}+\text { inflex }_{\mathbf{j}, \mathrm{b}}\right)}{\operatorname{regionDem}_{\mathrm{t}, \mathrm{z}}}\right] .
$$

Constraint Reformulation: in the new model the following constraint is equivalent:

$\operatorname{blockdev}_{\mathrm{h}, z, \mathrm{~b}, \mathrm{p}} \geqslant \operatorname{sign}_{\mathbf{p}} \times\left(\operatorname{blockTar}_{z, \mathrm{~b}, \mathrm{p}}-\frac{\sum_{\mathbf{j} \in \operatorname{Reg} z} \text { if }\left(\operatorname{tot}_{\mathbf{j}}=\mathrm{b}, \text { unitMW }_{\mathrm{t}, \mathrm{j}}, 0\right)}{\operatorname{regionDem}_{\mathrm{t}, z}}\right)$

where tot $_{j}=$ flex $_{j}+$ inflex $_{j}$. To linearize this constraint a binary decision variable seems to be necessary; which defeats the purpose as the binary variables are present in the original constraint.

\section{Constraint 4}

$$
\begin{aligned}
\text { flexdev }_{t, z, b, p} & \geqslant \operatorname{sign}_{\mathfrak{p}} \\
& \times\left(\text { flexTar }_{z, b, p}-\frac{\sum_{j \in \operatorname{Reg} z} \text { unitMW }_{t, j} \times \operatorname{flex}_{j, b}}{\text { blockTar }_{x, b, p} \times \text { regionDem }_{t, z}}\right) .
\end{aligned}
$$


Constraint Reformulation: in the new model, the following constraint is equivalent:

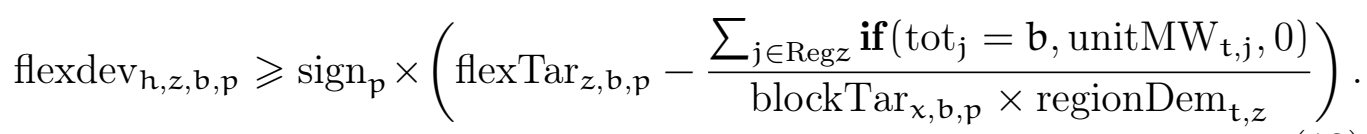

To linearize this constraint a binary decision variable seems to be necessary; which defeats the purpose as the binary variable is present in the original constraint.

Objective function The first three terms of the objective function need to be written in the following way:

totalcost

$$
\begin{aligned}
& =\sum_{j} \sum_{b}\left[\left(\operatorname{rop}_{j}+\operatorname{rcp}_{j, b}+\operatorname{rfp}_{j}+\text { duip }_{j, b}-\operatorname{frc}_{j}\right) \times \mathbf{i f}\left(\text { inflex }_{j}=b, 1,0\right)\right] \\
& +\sum_{j} \sum_{b}\left[\left(\operatorname{rop}_{j}+\operatorname{rcp}_{j, b}+\operatorname{rfrp}_{j}+\operatorname{rfp}_{j}\right) \times \mathbf{i f}\left(\text { flex }_{j}=b, 1,0\right)\right] \\
& +\sum_{j} \sum_{b}\left[\left(\text { duip }_{j, b}-\text { frc }_{j}\right) \times \mathbf{i f}\left(\text { flex }_{j}=b, 1,0\right) \times \frac{\text { flexProp }}{\text { flexProp }+ \text { addFlex }}\right]
\end{aligned}
$$

Suggestion The constraint programming solver in ILOG may be used to solve this alternative formulation as it can handle if then else type conditions.

\subsection{Second Model Reduction}

A second model reduction strategy is reported here. The primary idea is to assign demand units to blocks first, and then to specify whether it is flexible or 
inflexible separately. This in theory halves the number of the original binary variables required, for example, $2|J||B| \geqslant|J||B|+|J|$. Let $A_{j, b}$ be a binary decision variable for the assignment of a demand unit to a block and let $F_{j}$ be a binary variable to specify whether unit $j$ is to provide flexible AUFLS or not. The following conditions must be quantified in order to translate the model:

$$
\begin{aligned}
\text { flex }_{j, b} & =1, \quad \text { if } \quad A_{j, b}=1 \quad \text { and } F_{j}=1 ; \\
\text { llex }_{j, b} & =0, \quad \text { if } \quad A_{j, b}=0 \quad \text { or }\left(A_{j, b}=1 \quad \text { and } F_{j}=0\right) ; \\
\text { inflex }_{j, b} & =1 \quad \text { if } \quad A_{j, b}=1 \quad \text { and } F_{j}=0 ; \\
\text { inflex }_{j, b} & =0 \quad \text { if } \quad A_{j, b}=0 \quad \text { or } \quad\left(A_{j, b}=1 \quad \text { and } F_{j}=0\right) .
\end{aligned}
$$

The following non-linear equations are valid: $\operatorname{flex}_{j, b}=A_{j, b} F_{j} ;$ and inflex $j_{j, b}=$ $A_{j, b}\left(1-F_{j}\right)$.

Proof: If $F_{j}=1$ and $A_{j, b}=1$, then flex $x_{j, b}=1$ and inflex $x_{j, b}=0$ as required. If $F_{j}=0$ and $A_{j, b}=1$, then $f l e x_{j, b}=0$ and inflex ${ }_{j, b}=1$ as required. If $F_{j}=1$ and $A_{j, b}=0$, then flex $_{j, b}=0$ and inflex $x_{j, b}=0$ as required. If $F_{j}=0$ and $A_{j, b}=0$, then flex $_{j, b}=0$ and inflex ${ }_{j, b}=0$ as required.

The product of two binary variables can be linearized using the theory found in the AIMMS 3.13 modelling guide [12] in the section on products of variables. This approach allows flex $_{j, b}$ and inflex $_{j, b}$ to be converted to continuous variables:

$$
\begin{aligned}
\text { flex }_{j, b} & \leqslant A_{j, b}, \quad \text { flex }_{j, b} \leqslant F_{j}, \quad \text { flex }_{j, b} \geqslant F_{j}+A_{j, b}-1 \\
\text { inflex }_{j, b} & \leqslant A_{j, b}, \quad \text { inflex } \\
j, b & \leqslant 1-F_{j}, \quad \text { inflex } j_{j, b} \geqslant A_{j, b}-F_{j} .
\end{aligned}
$$


This is proof of the two relationships in Equations (15)-(16):

$$
\begin{aligned}
& F_{j}=1 \quad A_{j, b}=1 \quad 1 \leqslant \text { flex }_{j, b} \leqslant 1 \quad 0 \leqslant \text { inflex }_{j, b} \leqslant 0 \\
& \text { therefore } \text { flex }_{\mathbf{j}, \mathbf{b}}=1 \text { therefore inflex } \boldsymbol{j}_{\mathbf{j}, \mathbf{b}}=0 \\
& F_{j}=1 \quad A_{j, b}=0 \quad 0 \leqslant \text { flex }_{j, b} \leqslant 0 \quad-1 \leqslant \text { inflex }_{j, b} \leqslant 0 \\
& \text { therefore } \text { flex }_{j, b}=0 \text { therefore inflex } \boldsymbol{j}_{\mathbf{j}, \mathrm{b}}=0 \\
& F_{j}=0 \quad A_{j, b}=1 \quad 0 \leqslant \text { inflex }_{j, b} \leqslant 0 \quad 1 \leqslant \text { inflex }_{j, b} \leqslant 1 \\
& \text { therefore } \text { flex }_{j, b}=0 \text { therefore inflex } \mathbf{j}_{\mathbf{j}, \mathfrak{b}}=1 \\
& F_{j}=0 \quad A_{j, b}=0 \quad-1 \leqslant \text { inflex }_{j, b} \leqslant 0 \quad 0 \leqslant \text { inflex }_{j, b} \leqslant 0 \\
& \text { therefore } \text { flex }_{j, b}=0 \text { therefore inflex } \boldsymbol{j}_{\mathbf{j}, \mathrm{b}}=0
\end{aligned}
$$

The models constraints are changed in the following way.

Constraint $1 \sum_{\mathrm{b}}\left(\operatorname{flex}_{\mathfrak{j}, \mathrm{b}}+\right.$ inflex $\left._{\mathfrak{j}, \mathrm{b}}\right) \leqslant 1$ for all $\boldsymbol{j}$. Constraint Reformulation: $\sum_{b} A_{j, b} \leqslant 1$ for all $j$.

\section{Constraint 2}

$\operatorname{regdev}_{\mathrm{t}, z, \mathrm{p}} \geqslant \operatorname{sign}_{\mathrm{p}}$

$$
\times\left(\operatorname{region}_{\operatorname{Tar}_{z, \mathfrak{p}}}-\frac{\sum_{\mathbf{j} \in \operatorname{Reg} z} \sum_{\mathrm{b}} \text { unitMW }_{\mathrm{t}, \mathrm{j}}\left(\mathrm{flex}_{\mathfrak{j}, \mathrm{b}}+\operatorname{inflex}_{\mathbf{j}, \mathrm{b}}\right)}{\operatorname{regionDem}_{\mathrm{t}, z}}\right) .
$$

Constraint Reformulation:

$$
\begin{aligned}
\operatorname{regdev}_{h, z, p} & \geqslant \operatorname{sign}_{p} \times\left(\operatorname{region} \operatorname{Tar}_{z, p}-\frac{\sum_{j \in \operatorname{Reg} z} \text { unitMW }_{h, j} A_{j, b}}{\operatorname{regionDem}}\right) \\
\operatorname{tot}_{j}, z & \leqslant M \alpha_{j} \text { for all } j, \\
\operatorname{tot}_{j} & \geqslant 1+M\left(\alpha_{j}-1\right) \text { for all } j .
\end{aligned}
$$

\section{Constraint 3}

blockdev $_{\mathrm{t}, z, \mathrm{~b}, \mathrm{p}} \geqslant \operatorname{sign}_{\mathrm{p}}$

$$
\times\left(\operatorname{blockTar}_{z, b, p}-\frac{\sum_{j \in \operatorname{Reg} z} \text { unitMW }_{t, j}\left(f l e x_{j, b}+\text { inflex }_{j, b}\right)}{\operatorname{regionDem}_{t, z}}\right) .
$$


Constraint Reformulation:

blockdev $_{\mathbf{h}, z, \mathrm{~b}, \mathrm{p}} \geqslant \operatorname{sign}_{\mathfrak{p}} \times\left[\operatorname{blockTar}_{z, \mathrm{~b}, \mathrm{p}}-\frac{\sum_{\mathbf{j} \in \operatorname{Reg} z} \text { unitMW }_{\mathrm{t}, \mathrm{j}} A_{\mathrm{j}, \mathrm{b}}}{\operatorname{regionDem}_{\mathrm{t}, z}}\right]$.

\section{Constraint 4}

$$
\begin{aligned}
& \text { flexdev }_{\mathfrak{t}, z, \mathbf{b}, \mathfrak{p}} \geqslant \operatorname{sign}_{\mathrm{p}} \\
& \times\left(\operatorname{flexTar}_{z, b, p}-\frac{\sum_{j \in \operatorname{Reg} z} \text { unitMW }_{t, j} \times \text { flex }_{\mathbf{j}, \mathrm{b}}}{\text { blockTar }_{x, \mathrm{~b}, \mathrm{p}} \times \operatorname{regionDem}_{\mathrm{t}, \mathrm{z}}}\right) .
\end{aligned}
$$

Constraint Reformulation: No change.

\subsection{Data Reduction}

In order to solve the decision problem more quickly, model reduction activities are important. However, it makes as much sense to perform data reduction, as that activity will also simplify the solution process. Two methods of data reduction could be useful. One is to use stratified sampling techniques on the demand data, and the other is to remove feeders that have a very low probability of being in the solution due to high cost or variability.

\subsubsection{Stratified Sampling}

Some specific features are evident in Figures 2 and 3. There is a difference in level of overall demand between summer and winter. There is also evidence in Figure 2 of a lower demand in general around the Christmas period, between hours 12000 and 14000. These features are common throughout the three regions that the grid is divided into. Region 1 contains the Auckland area. From Figure 3, there is a difference between weekdays and weekends in the general level of demand, with weekends exhibiting lower demand in general. 


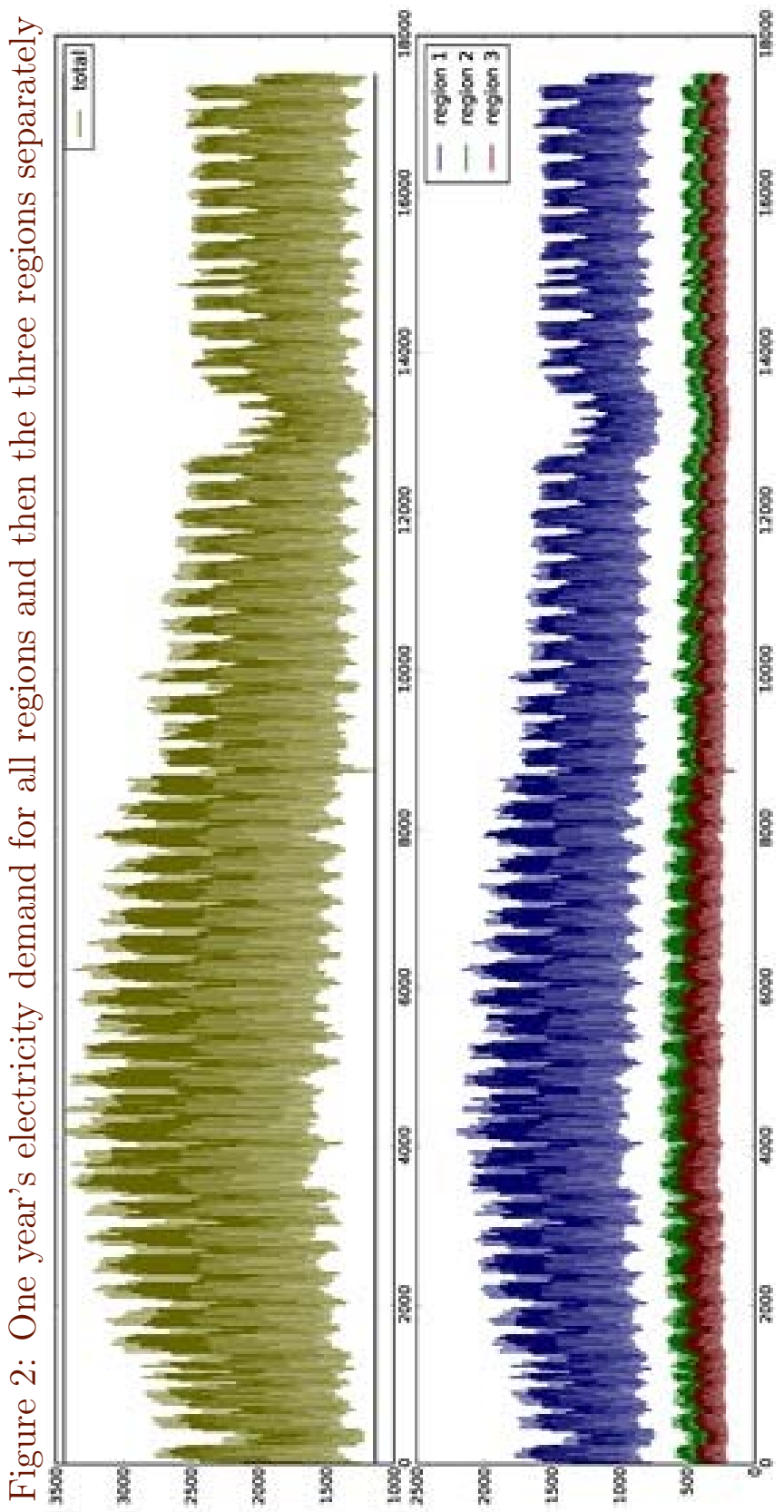




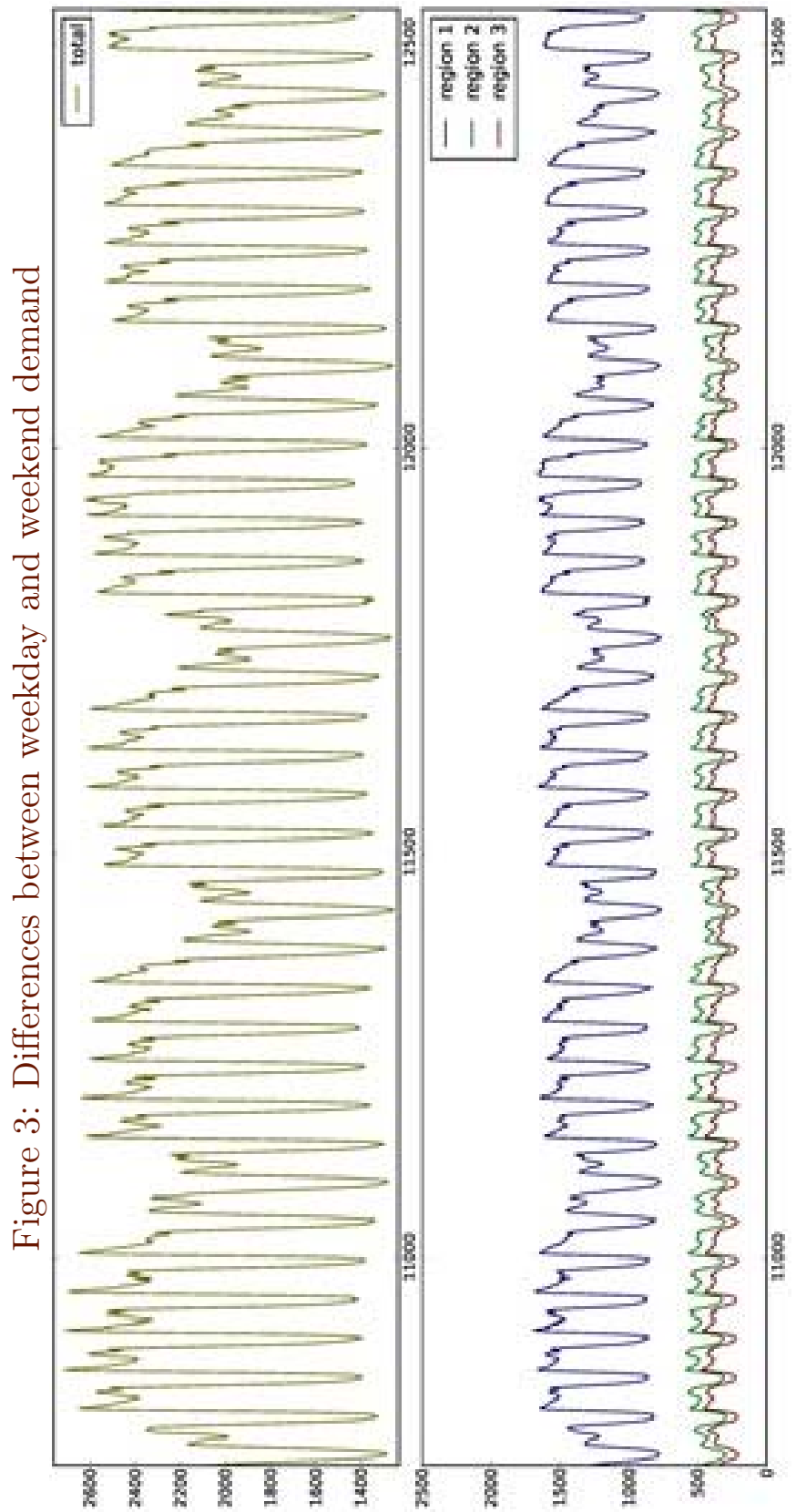


There is also the difference between the higher daytime demand with the lower night-time demand. As well as these features, there are two intraday peaks, one in the morning and one late in the afternoon, early evening. A sensible strategy for selecting a sample of half hour demand data to perform the optimisation on is to select at random within these different strata. The numbers selected from each stratum would be in proportion to how many half hours of that type there are in the year. For instance, there are 100 weekend days, excluding the two weekends in the Christmas period, so there should be $100 \times 48 / 17520$ of the total number of hours selected to be from weekend days in the final sample

\subsubsection{Ranking Feeders}

There are two reasons why a feeder would have a low probability of being selected in the final set to have relays installed or armed. One is that the demand on that feeder is highly variable, meaning that one could not depend on that feeder to provide the contribution to lowering the demand for the whole period that it might take to repair any malfunction. The other reason is that the loss of load cost might be prohibitively high, a problem since a significant component of the objective is to minimise loss of load. Thus we calculated the variability for each feeder in the following manner. At each half hour, we calculated the contribution that the feeder made to the demand in that region. We used the regional contribution since there are constraints that relate to regional imperatives. Call this contribution $c_{i j}$, where $i$ is the time and $\boldsymbol{j}$ is the feeder. Then form the coefficient of variation $v_{j}$ for the contribution of each of these feeders over the year, this being the standard deviation divided by the mean.

If we define the cost of the loss of load in each feeder if it has to be shut down as $L_{j}$, then let us denote the Index of Inclusion of the feeder to be the product of the coefficient of variation and loss of load, $I_{j}=v_{j} L_{j}$. Figure 4 gives the histogram of this index for the three regions. The feeders with high values of $I_{j}$ will have a low probability of being members of the final solution 
Figure 4: Cumulative histogram of the index for ranking feeders

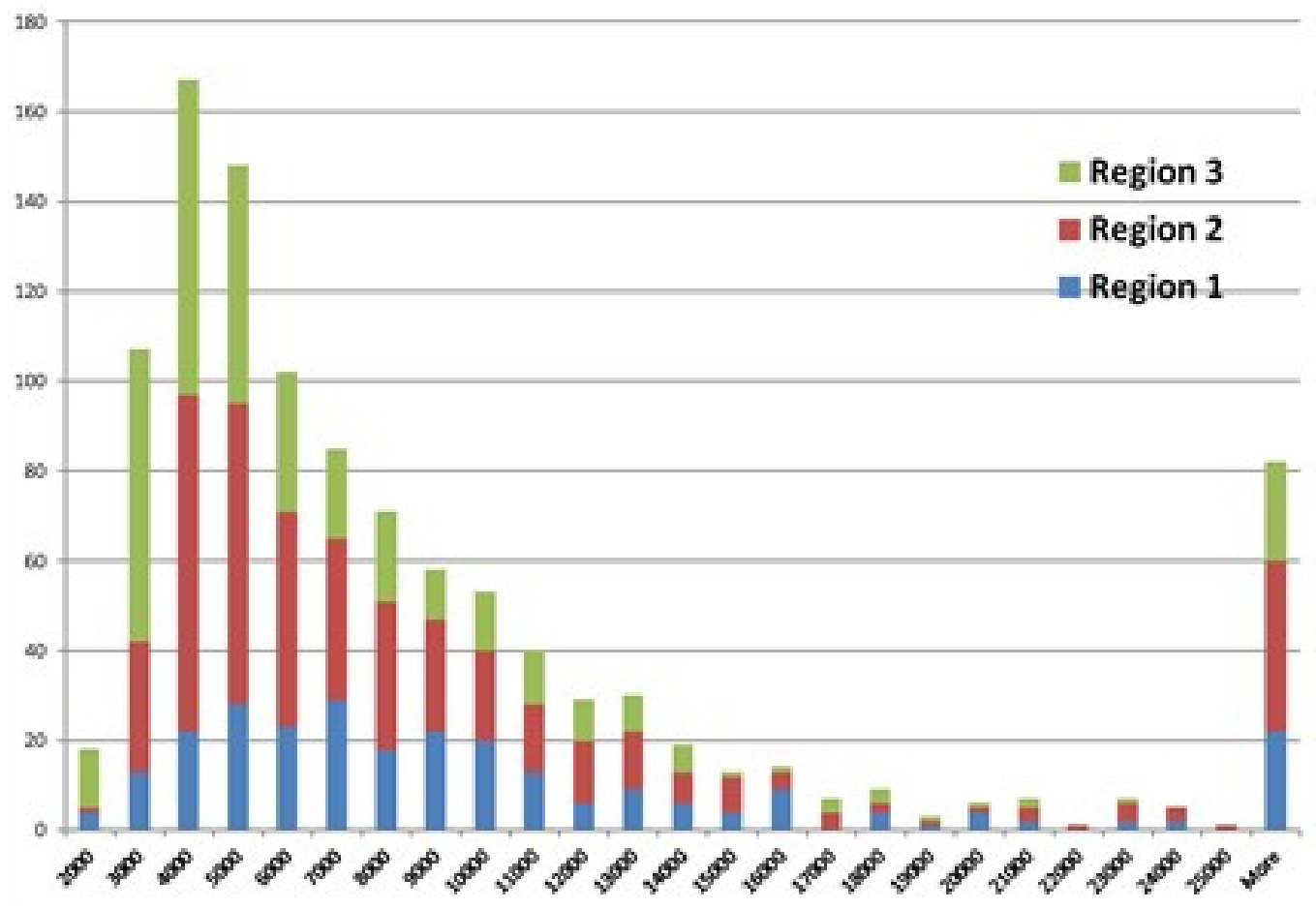

set. Thus it would be opportune to eliminate them from consideration as potential locations for relays. Thus we would discard the feeders in the large spike at the right end of the histogram, but potentially also any in the long tail, for instance perhaps with a values of $I_{j}>16,000$. How one might test whether this is reasonable is to solve the problem with all of those feeders discarded, and then try with the next category of feeders added back in. If there is little impact on the solution set, then this cut-off is appropriate. If this were the case, then perhaps one could discard another group of feeders and see if this affected the solution. 


\section{$5 \quad$ Bi Objective Model}

A model that considers a historical time horizon using actual electricity consumption data is presented here. It was formulated by Josh Chopin, Oliver Czibula, and Joey Fung. Two conflicting objectives were considered:

1. minimising the total cost of selecting feeders for flexible or inflexible relays over the considered time horizon; and

2. minimising the total violations of the desired values $10 \%$ and $6 \%$ over the considered time horizon.

This section also presents a methodology which utilises the model to analyse the trade-off over the considered time horizon, between the cost of selecting feeders for relays versus the number of violations of the desired load values.

\subsection{Model and Methodology}

The model uses as input the historical electricity consumption data and the cost of selecting each feeder for each block as either a flexible relay or an inflexible relay. A set of user controlled parameters determine the upper and lower thresholds for the electricity load of each flexible and inflexible block. Thus, a block is said to violate its desired load value of $10 \%$ or $6 \%$ if the total electricity load of its flexible and/or inflexible electricity load are above its upper threshold or below its lower threshold. Another parameter controlled by the end user is the upper limit on the total number of violations over the planning horizon. The number of violations at any time point is the number of blocks for which the desired load value is violated at the time point. Thus, since there are four blocks, the number of violations at each time point is at most four. The model aims to find a minimal cost assignment of feeders to relay blocks such that the total number of violations over the time horizon does not exceed the upper limit set by the end user. The parameters, variables and sets of the model are summarised in Tables 2, 3 and 4 . 
Table 2: Parameters

Parameter Description

$c_{j, b}^{F} \quad$ The cost of assigning feeder $j$ to block $b$ as an flexible relay.

$c_{j, b}^{1} \quad$ The cost of assigning feeder $\boldsymbol{j}$ to block $b$ as an inflexible relay.

$\rho_{j, t} \quad$ The consumption of electricity for feeder $j$ at time $t$, expressed as a fraction of total electricity consumption at time $t$.

$\mathrm{F}_{\mathrm{b}}^{+} \quad$ The upper threshold for flexible relays in block $\mathrm{b}$, determined by the desired value plus the error threshold.

$\mathrm{F}_{\mathrm{b}}^{-} \quad$ The lower threshold for flexible relays in block $\mathrm{b}$, determined by the desired value minus the error threshold.

$\mathrm{I}_{\mathrm{b}}^{+} \quad$ The upper threshold for inflexible relays in block $\mathrm{b}$, determined by the desired value plus the error threshold.

$\mathrm{I}_{\mathrm{b}}^{-} \quad$ The lower threshold for inflexible relays in block $\mathrm{b}$, determined by the desired value minus the error threshold.

$\mathrm{V} \quad$ The user-determined upper limit to the total violations.

C The upper limit to the total cost.

\section{Table 3: Variables}

\begin{tabular}{|c|c|}
\hline Variables & Description \\
\hline$f_{j, b}$ & $\begin{array}{l}\text { Binary variable. Equals to } 1 \text { if feeder } \boldsymbol{j} \text { is assigned to block b } \\
\text { as a flexible relay, } 0 \text { otherwise. }\end{array}$ \\
\hline$i_{j, b}$ & $\begin{array}{l}\text { Binary variable. Equals to } 1 \text { if feeder } \boldsymbol{j} \text { is assigned to block b } \\
\text { as an inflexible relay, } 0 \text { otherwise }\end{array}$ \\
\hline$v_{t, b}$ & $\begin{array}{l}\text { Binary variable. Equals to } 1 \text { if flexible relays, inflexible relays } \\
\text { or both types of relays of block b is above or below its desired } \\
\text { load value at time } t \text {. }\end{array}$ \\
\hline
\end{tabular}


Table 4: Sets

\begin{tabular}{cl}
\hline Sets & Description \\
\hline J & The set of all feeders. \\
$\tilde{J}$ & A subset of J which are pre?selected, that is, must be assigned \\
& to a block. \\
B & The set of all blocks, that is $\mathrm{B}=\{1,2,3,4\}$. \\
$\mathrm{T}$ & The set of all time points. \\
\hline
\end{tabular}

The proposed solution methodology first uses the model to minimise the total cost of selecting feeders for flexible and inflexible relays, subject to not exceeding the upper limit to the number of violations over the considered time horizon. Letting this cost be referred to as the minimal cost, the methodology then uses the model to minimise the total number of violations over the considered time horizon, subject to the new total cost not exceeding some threshold of the minimal cost obtained in the first run. This cost threshold can be specified by the end user, and a number of cost thresholds can be specified for consideration. Thus the result of the trade-off analysis is that for each cost threshold, the minimum number of total violations over the considered time horizon is given.

The mixed integer linear programming (MILP) model is now presented using the variables, sets and parameters described in Tables 2 to 4, respectively. This MILP model considers two conflicting objectives. Each objective has a corresponding constraint that is utilised only with that particular objective. Thus, in the first instance, when the objective is to minimise the total cost of selecting feeders for flexible and inflexible relays subject to the total violations not exceeding the user given maximum, the objective function and its corresponding constraint are given in (21) and (22), respectively:

$$
\begin{aligned}
& \min \sum_{j \in J} \sum_{b \in B}\left(c_{j, b}^{F} f_{j, b}+c_{j, b}^{I} i_{j, b}\right) ; \\
& \sum_{t \in T} \sum_{b \in B} v_{t, b} \leqslant v .
\end{aligned}
$$


In the latter instances, when the objective is to minimise the total number of violations subject to the total cost not exceeding a user given cost threshold, the objective function and its corresponding constraint are given in (23) and (24), respectively:

$$
\begin{aligned}
& \min \sum_{t \in T} \sum_{b \in B} v_{t, b} ; \\
& \sum_{j \in J} \sum_{b \in B}\left(c_{j, b}^{F} f_{j, b}+c_{j, b}^{I} i_{j, b}\right) \leqslant C .
\end{aligned}
$$

The parameters $V$ and $C$ respectively are the upper limits on the number of violations and the total cost. Unlike constraints (22) and (24), the next two constraints are utilised in either instance of the model:

$$
\begin{array}{ll}
\sum_{b \in B}\left(f_{j, b}+i_{j, b}\right)=1 & \text { for all } j \in \tilde{J} ; \\
\sum_{b \in B}\left(f_{j, b}+i_{j, b}\right) \leqslant 1 \quad \text { for all } j \in(J-\tilde{J}) .
\end{array}
$$

Each pre-selected feeder in $\tilde{J}$ must be assigned to one of the four blocks, whilst each remaining feeder can be assigned to at most one block as either flexible or inflexible. The following constraints measure whether the electricity load of the flexible relays in each block $b$ at each time $t$ is below the lower threshold $\mathrm{F}_{\mathrm{b}}^{-}$or is above the upper threshold $\mathrm{F}_{\mathrm{b}}^{+}$for the block:

$$
\begin{aligned}
& \sum_{j \in J}\left(\rho_{j, t} f_{j, b}\right) \geqslant\left(1-v_{t, b}\right) F_{b}^{-} \quad \text { for all } b \in B, t \in T ; \\
& \sum_{j \in J}\left(\rho_{j, t} f_{j, b}\right) \leqslant F_{b}^{+}+v_{t, b}\left(\sum_{j \in J} \rho_{j, t}-F_{b}^{+}\right) \quad \text { for all } b \in B, t \in T .
\end{aligned}
$$

Observe that when $v_{\mathrm{t}, \mathrm{b}}=0$ then $\mathrm{F}_{\mathrm{b}}^{-} \leqslant \sum_{\mathrm{j} \in \mathrm{J}}\left(\rho_{\mathrm{j}, \mathrm{t}} \mathrm{f}_{\mathrm{j}, \mathrm{b}}\right) \leqslant \mathrm{F}_{\mathrm{b}}^{+}$. Alternatively if $v(t, b)=1$, then the right hand side of (25) becomes $\sum_{j \in J} \rho_{j, t}$ and hence is 
not restrictive. Similarly, for inflexible relays:

$$
\begin{aligned}
& \sum_{j \in J}\left(\rho_{j, t} i_{j, b}\right) \geqslant\left(1-v_{t, b}\right) I_{b}^{-} \quad \text { for all } b \in B, t \in T \\
& \sum_{j \in J}\left(\rho_{j, t} i_{j, b}\right) I_{b}^{+}+v_{t, b}\left(\sum_{j \in J}\left(\rho_{j, t}-I_{b}^{+}\right) \quad \text { for all } b \in B, t \in T .\right.
\end{aligned}
$$

\subsection{Preliminary Results}

The model was computationally tested with real data supplied by Transpower. There were 1194 feeders on the network where relays may be placed. For each feeder, a numerical value describing the electricity demand for half hourly intervals for a period of one year was supplied. The ordered bi-criteria model was solved for a number of contiguous time intervals: one day, one week, and four weeks. For the primary objective of minimising cost, we set the upper bound of $\sum_{t \in T} \sum_{b \in B} \nu_{t, b}$ to be no greater than $|\mathrm{T}||\mathrm{B}| / 2$. For the secondary objective of minimising the total number of violations, we permitted the optimal cost from the secondary objective to deteriorate by at most $10 \%$. The optimal solution from the primary model was used as an initial solution for the secondary model.

Figure 5 shows the decrease in the number of violations when permitting an increase in the cost, for different number of time points.

\subsection{Pareto Analysis}

The model proposed in this section is bi-objective and can be solved as such without the need for a two stage process. Without loss of generality, the goal of multi objective optimization is to find solutions that are considered equally good for all objectives or in technical terms to identify the set of non-dominated "Pareto" optimal solutions. Pareto optimality is a state in 
Figure 5: Preliminary Results

\begin{tabular}{rrrr}
\hline $\begin{array}{l}\text { \#Time } \\
\text { points }\end{array}$ & Cost $(\mathrm{k} \$)$ & $\begin{array}{l}\text { \#Violations } \\
\text { Solve } \\
\text { time } \\
\text { (secs) }\end{array}$ \\
\hline 48 & 6,615 & 192 & 0.9 \\
48 & 6,826 & 96 & 323.7 \\
96 & 6,648 & 384 & 1.9 \\
96 & 6,806 & 192 & 907.0 \\
\hline
\end{tabular}

which it is impossible to make any one objective better without making at least one objective worse. In other words there is no other point that improves at least one objective function without detriment to another function. Several well-known approaches exist for solving these multi-objective models. These include, for instance, the $\epsilon$-constraint method (ECM), weighted sum method (WSM), and normal boundary (NBI) method. Many of these techniques attempt to identify a set of candidate solutions, from which a single solution is then chosen. Most of these methods also produce only one point on the Pareto frontier per iteration. The Pareto frontier can be pre-computed in order to facilitate the evaluation of different preferences at a later time. Otherwise, it is possible to solve for the best solution directly. That course of action is less often taken as it results in an optimisation problem with a non-linear objective criterion, involving traditionally some measure of distance from the utopia point. The utopia point is a fictional solution that obtains the best possible value for each objective. To compare the different objective functions, the values of $\Phi_{k}(x)$ are normalised in the following way: $\tilde{\phi}_{k}(x)=\left(\phi_{k}(x)-\underline{\phi}_{k}\right) /\left(\bar{\phi}_{k}-\underline{\phi}_{k}\right)$. To judge the merit of solutions on the Pareto frontier the distance from the utopia point (i.e., the best possible solution) is then computed. Figure 6 summarises key features of bi-objective optimisation. 
Figure 6: Solution quality changes when moving along the Pareto frontier
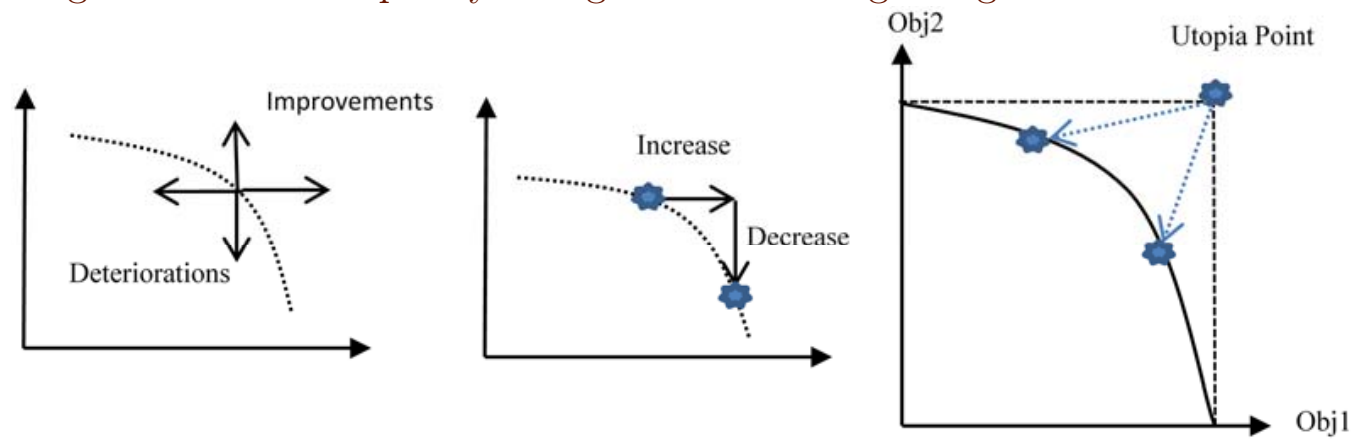

\section{Solution Techniques}

\subsection{Optimisation Simulation}

In order to create a solution that is robust across the entire year, that is for different system demands, an optimisation-simulation approach could be appropriate. Here simulation refers to the analysis of the system's performance for a variety of different scenarios whereby frequency variations and fluctuations occur. This could be arbitrarily selected; however, historical data exists that could be used. The use of historical data was identified as being more appropriate as it describes what actually happened. Note that the expected cost over say $\mathrm{N}$ simulations is required every time a solution is evaluated. The optimisation technique remains the same otherwise. As an alternative to optimisation simulation a bi-objective approach is also appropriate. The first objective is to minimise cost, but the second is to maximise robustness. The bi-objective model can be solved using a Pareto analysis such as the $\epsilon$-constraint method. This bi-objective approach requires a measure of robustness to be specified. Burdett \& Kozan [2] reported the following as the most accepted definition for robustness.

Definition 1. A solution is defined as robust if it is insensitive to delays, 
deviations and uncertain events. The most robust solution is most insensitive. A solution can only be robust to a specified tolerance.

\subsection{Meta Heuristic Approach}

Meta heuristic techniques are strategies that guide search processes. Their goal is to efficiently explore the search space in order to find near-optimal solutions. These techniques are computationally fast, and scale well as the problem size is increased. Exact techniques often do not scale well and can become intractable very quickly. There is a wide variety of meta-heuristics such as Simulated Annealing (SA), Genetic Algorithms (GA), Evolutionary Algorithms (EA), and Tabu Search (TS). Table 5 briefly summarises their merits.

In order to apply these algorithms a number of decisions have to be made.

Decision 1 Use binary decision variable, integer decision variables, or something more novel.

Decision 2 To enforce the constraints of the model there are three options:

- Penalise constraint violations in the objective function;

- Apply solution correction procedures to return solution to feasibility;

- Restrict moves (i.e., perturbations) that cause constraint violations.

For this decision problem, integer variables are a better choice than binaries. The integer decision variables can be treated as continuous and then truncated to the nearest integer. SA and TS are well suited; however, a special EA called Differential Evolution (DEA) was found to be most successful. An alternative is to use a natural "discrete" representation of the solution. For example, as each block has a list of assigned demand units, the different lists are the 
Table 5: Summary of different techniques

\begin{tabular}{lll}
\hline Algorithm & Advantage & Disadvantage \\
\hline Genetic Algorithm & GA most suited when & Population based ap- \\
and Evolutionary & there are binary deci- proaches so may be \\
Algorithm & sion variables. Stan- slow. Performance may \\
& dard crossover and bit be poor because fine \\
& flipping may be used. tuning is more difficult \\
& EA is generally superior to do. \\
& to GA because a "natu- \\
& ral" solution representa- \\
& tion can be used. DEA \\
& is very good when the \\
& decision variables are \\
& continuous. \\
Strong all round tech- Produces only a single \\
Simulated Annealing \\
niques. Fastest and solution of high quality \\
most adaptable algo- \\
rithms.
\end{tabular}

solution. Hence perturbation of the solution simply involves moving units to different blocks or else exchanging units between different blocks. This is shown in Figure 7.

An alternative is to give each demand unit a type: for example $f$, flex; $i$, inflex; $n$, no type. Hence switching the type is a secondary perturbation operator (Figure 8).

For this decision problem a Local Search (LS) algorithm was implemented by Alan Lee. In that approach a starting solution is first generated. That solution is refined via simple perturbations. The current solution is changed if and only if the new solution is strictly better. When local optimality is reached, the current solution is randomly perturbed and the process is started again. The whole process is terminated according to a time based stopping criteria. 
Figure 7: Solution representation and a shift and exchange perturbation

\begin{tabular}{|c|c|c|c|c|c|}
\hline Category & $\begin{array}{c}\text { Block 0 } \\
\text { (dummy) }\end{array}$ & Block 1 & Block 2 & Block 3 & Block 4 \\
\cline { 1 - 5 } & \multirow{2}{*}{$1,3,13,14,15$} & & 4 & 7 & 10,11 \\
\cline { 1 - 3 } & & 7 & 8 & 9,6 & 12 \\
\hline Inflexible & & & & & \\
\hline
\end{tabular}

Figure 8: Alternative solution representation

\begin{tabular}{|c|c|c|c|c|}
\hline $\begin{array}{c}\text { Block 0 } \\
\text { (dummy) }\end{array}$ & Block 1 & Block 2 & Block 3 & Block 4 \\
& & & & \\
\hline $1(\mathrm{n}), 3(\mathrm{n}), 13(\mathrm{n}), 14(\mathrm{n}), 15(\mathrm{n})$ & $2(\mathrm{f}), 7(\mathrm{i})$ & $4(\mathrm{f}), 8(\mathrm{i})$ & $5(\mathrm{f}), 9(\mathrm{i}), 6(\mathrm{i})$ & $10(\mathrm{f}), 11(\mathrm{f}), 12(\mathrm{i})$ \\
\hline
\end{tabular}

\subsection{Heuristic Approach}

The aforementioned meta-heuristics require a single starting solution or a population of different starting solutions. In order to accomplish this, constructive heuristic techniques are required. In essence these are basic rule based algorithms that allow a solution to be constructed quickly. They are rather "myopic" (narrow sighted), and typically are greedy because they make lots of locally optimal decisions. At MISG a strategy was proposed and tested, whereby demand units are ranked according to a specified score. This ranking is iterated through, and demand units are assigned as they are visited, for example to satisfy each blocks percentage target. The blocks are considered one by one, starting from the first. Small under or over assignment 
is penalised in the objective function. To obtain other solutions, the ranking is perturbed. The best solution is chosen from the list of candidate solutions. A ranking (scoring) mechanism was proposed. There are two components involved. First, the contribution of each feeder at each time to the region's load or demand is computed. Second, the coefficient of variation for each feeder is found. The demand weighted cost of loss of load for each feeder is then computed. The product of these values is then computed and the feeders are sorted.

\subsection{Solution Similarity and Repeatability}

When the solution process is not exact, that is it does not produce the optimal solution, then different solution configurations may be obtained every time that method is applied. From a practical viewpoint that is not entirely acceptable for Transpower who would like repeatable solutions. This issue is rather complex and requires further research. At this stage some comparison metrics have been proposed. The idea is that these metrics quantify how similar two specified solutions are to each other. The metrics are as follows and are self-explanatory:

$$
\begin{aligned}
& \text { scoreflex }=\frac{\mid\left\{(\mathfrak{j}, \mathbf{b}): \text { flex }_{\mathbf{j}, \mathbf{b}}=\mathrm{flex}_{\mathbf{j}, \mathbf{b}}^{\prime}\right\} \mid}{|J||\mathrm{B}|} ; \\
& \text { scoreinflex }=\frac{\mid\left\{(j, b): \text { inflex }_{j, b}=\text { inflex }_{\mathbf{j}, \mathrm{b}}^{\prime}\right\} \mid}{|J||B|} ; \\
& \text { score }=\frac{\text { scoreflex }+ \text { scoreinflex }}{2} ; \\
& \text { score }=\frac{\left|\left\{(j, b): A_{j, b}=A_{j, b}^{\prime}\right\}\right|}{|J||B|} .
\end{aligned}
$$

An approach that identifies all optimal solutions can be applied. This task is called multi-modal optimization 


\section{Conclusions}

This article focussed on automatic under frequency load shedding practices used in New Zealand's power system. We sketched recent research trends, analysed the existing model, proposed new models, and identified a variety of alternative solution techniques.

In conclusion we identified that solution robustness can be verified by analysing the system performance under historical events.

Solution repeatability was specified as an important issue. For example, obtaining vastly different solutions by different solution techniques or by each application of a solution technique is undesirable. A repeatable solution is needed that is either optimal, or close to optimal. We identified that a metric of solution similarity is needed, for example to compare how different the obtained solution is from current practice.

Acknowledgments We thank Conrad Edwards of Transpower for the chance to work on this problem at MISG. The following people worked on this project during MISG and we acknowledge their ideas and their participation: Amie Albrecht, Bayan Bevrani, Josh Chopin, Luigi Cirocco, Oliver Czibula, Joey Fung, Alan Lee, Sam Liu, Mahmoud Masoud, Sam Patterson, Robin Pearce, Martin Peron, Danuya Rose.

\section{References}

[1] Arroyo J. M. and Galiana F. D. Energy and reserve pricing in security and network constrained electricity markets. IEEE Transactions on Power Systems, 20(2):C634-C643, 2005. M9, M10 
[2] Burdett R. and Kozan, E. Performance profiling for predictive train schedules. Journal of Rail Transport Planning and Management, 4:C98-C114, 2014. doi:10.1016/j.jrtpm.2014.11.002. M30

[3] Hiscock J., Hiscock N. and Kennedy A. Advanced voltage control for networks with distributed generation. 19th International Conference on Electricity Distribution. Vienna, 21-24 May, 2007. M8

[4] Just S. and Weber C. Pricing of reserves. Energy Economics, 30:C3198-C3221, 2008. M4, M8

[5] El-Khattam W., Bhattacharya K., Hegazy Y. and Salama M. M. A. Optimal investment planning for distributed generation in a competitive electricity market. IEEE Transactions On Power Systems, 19(3). M9

[6] Kusano N. New trends in protection relays and substation automation systems in Japan. Transmission and Distribution Conference and Exhibition 2002: Asia Pacific. IEEE/PES, 1, 624-628. M8

[7] Lopes J. A., Hatziargyriou N., Mutale J., Djapic P. and Jenkins, N. Integrating distributed generation into electric power systems: A review of drivers, challenges and opportunities. Electric Power Systems Research, 77, 1189-1203. M4

[8] Thomson M. Automatic voltage-control relays and embedded generation. Power Engineering Journal, April, 71-76. M8

[9] Wong S. and Fuller D. J. Pricing energy and reserves using stochastic optimization in an alternative electricity market. IEEE Transactions on Power Systems, 22(2), 631-638. M4, M9

[10] Transpower AUFLS Summary Card. WL 13309 https:

//www.systemoperator.co.nz/sites/.../AUFLS_Summary_Card.pdf M5

[11] Transpower Electricity reserve technical requirements schedule-ERTWG https://www.systemoperator.co.nz/sites/ 
default/files/bulk-upload/documents/ERTWG\%20Trial\% 20Procurement $\% 20$ Schedule\%20Guide.pdf M10

[12] AIMMS AIMMS 3.13 - AIMMS Modeling Guide - Integer Programming Tricks. http://www.aimms.com M17

\section{Author addresses}

1. Robert Burdett, School of Mathematical Sciences, Queensland University of Technology, Brisbane, Queensland Australia. mailto:r.burdett@qut.edu. au orcid:0000-0001-8930-1552

2. Conrad Edwards, Transpower Ltd. mailto:Conrad.Edwards@transpower.co.nz

3. John Boland, School of ITMS, University of South Australia, South Australia 5095, Australia.

mailto: john.boland@unisa.edu.au 